\title{
The second victim requires more than Medice Cura Te Ipsum
}

\author{
Martin A. Koyle, MD, MSc'; Michael E. Chua, MD'; Tamizan Kherani, MD, MSc'; Nichole Pereira, BScN, MN; \\ Kurt Heiss, $M D^{4}$
}

'Hospital for Sick Children, University of Toronto Department of Surgery and Institute of Health Policy, Management, and Evaluation, Toronto, ON, Canada; ${ }^{2}$ Stollery Children's Hospital, University of Alberta, Department of Pediatrics, Edmonton, AB, Canada; ${ }^{3}$ Queen's University, Kingston, ON, Canada; ${ }^{4}$ Children's Healthcare of Atlanta, Emory University School of Medicine, Atlanta, GA, United States

Cite as: Koyle MA, Chua ME, Kherani T, et al. The second victim requires more than Medice Cura Te Ipsum. Can Urol Assoc J 2021;15(6Suppl1):S40-2. http://dx.doi.org/10.5489/cuaj.7229

\section{Abstract}

Burnout has attained epidemic proportions in all reaches of society. Only recently, has its impact in healthcare become a burning platform. Second victim syndrome, a consequence of an unforeseen adverse event, often precipitated by an error, can lead to a post-traumatic stress-like reaction, that is unique to healthcare workers. Often, the second victim suffers in silence, forced to rely on resilience. Peer support has been demonstrated to be beneficial in assisting healthcare workers in recovering from both burnout and second victim syndrome. Institutions and organizations must be more influential and responsive in supporting physicians and other healthcare workers in need.

\section{Introduction}

A 29-year-old male born with a solitary kidney undergoes exploration for a $6 \mathrm{~cm}$ heterogeneous lower pole renal mass. The patient undergoes laparoscopic heminephrectomy, without clamping of the hilar vasculature. Significant bleeding occurs, which requires conversion to an open approach, at which time the renal vessels are clamped. The patient is resuscitated and stabilized in the operating room, requiring 6 units of packed red cells and other blood products. When the clamp is released from the renal vessels, the remaining renal parenchyma appears non-perfused, and Doppler of the renal artery demonstrates no flow. A vascular surgeon is consulted, and an intimal tear is found and repaired with restoration of flow. The patient is placed on anticoagulation and taken to the intensive care unit. Over the next several days, although urine output eventually occurs, the patient becomes dialysis dependent. The urologist asks for a leave of absence, but never returns to work.

\section{The second victim syndrome}

Healthcare has become both increasingly complex and stressful. Despite the best intentions of physicians and others involved in healthcare, unforeseen incidents and errors occur, leading to patient harm, the first victim (FV). Medical errors are the third leading cause of death in the United States, behind cancer and trauma. ${ }^{1}$ Second victims (SV) are healthcare workers who become traumatized by any event that may have predisposed to the occurrence of a FV situation. ${ }^{2}$ Such incidents can escalate even further, beyond that of the FV and SV, with institutional reputation impact, litigation, and potential harm to other patients being cascading results. The example in the case depicted in the introduction demonstrates that despite best intentions, knowledge, and skill, when a serious patient event occurs, harm can cascade beyond the patient to the provider him/herself.

Knowing that humans are not infallible, and healthcare workers participate within imperfect systems, the Institute of Medicine published To Err is Human in 2000. ${ }^{3}$ The Institute of Healthcare Improvement $(\mathrm{IHI})$ developed the patient-focused triple aim, with the goals of improving patient experience and the health of the population as a whole, while reducing healthcare costs per capita. Lacking in the triple aim, however, was a provider component. This resulted in the concept of a quadruple aim, which added a fourth dimension and goal to the initial IHI objectives: to improve the experience of those who provide care. ${ }^{4}$

Burnout is not unique to healthcare providers, as opposed to the SV syndrome. The latter is an acute stress disorder, with similar manifestations to post-traumatic stress disorder (PTSD) that occurs in healthcare workers, which can precipitate burnout and its consequences, and vice versa. ${ }^{2}$ It is likely that as many as half of healthcare providers will be SVs at some point in their career. ${ }^{5}$

\section{The course in SV syndrome}

Scott and colleagues from the University of Missouri suggest that most SVs will follow a fairly predictable course of recov- 
ery following the instigating sentinel event. ${ }^{6}$ The recovery trajectory is comprised of six stages of variable lengths: 1 ) chaos and confusion; 2) intrusive reflections; 3 ) restoring personal integrity; 4) enduring the inquisition; 5) obtaining personal first aid; and 6) moving on. In the first two stages, the healthcare provider experiences moral distress, attempts to comprehend the event, and may re-live it in his/her mind. Stage 3 is of significance, as this is the time when the provider may self-reflect or seek external support to understand the system-level influences of an incident and his/her role. Stage 4 can be particularly intimidating, as the SV deals with the ramifications that the event might have upon one's career, such as litigation threats and licensing sanctions, as well as potential financial sequelae. This leads to the fifth stage, where safety is sought, and the provider may feel particularly insecure about who might be safe to confide in. Lastly, the final stage may have three consequences pertaining to moving on - the two extremes being thriving as a wiser, safer clinician, or dropping out, with those in the middle merely surviving. Of course, the scope of the incident and the resilience of the involved individual impact each of these stages. Every clinician mourns a failure differently. Integral to recovery outcome is the culture in which the individual works and the response of the system or organization. ${ }^{7}$ It is more than imperative that SVs and clinicians experiencing burnout are provided with ongoing support for quality and patient safety issues. Not only are incident and error rates increased in these individuals, the practice of defensive medicine and their related healthcare costs also tend to escalate. ${ }^{8,9}$

\section{Supporting the SV}

Although the numbers are increasing, most institutions do not have a culture that prevents and ultimately provides recovery support to SVs and those who suffer from burnout. Physicians are particularly fearful of appearing vulnerable and too often "suffer in silence" because of concerns related to confidentiality. Data is accruing that suggests that peer support, by properly trained colleagues and networks, rather than only confiding in family and friends, is most beneficial during the stages of recovery. ${ }^{10,11} \mathrm{Scott}$ and the Missouri group have advocated for the creation of rapid response teams (RRT) that are mobilized and involved early after an incident, knowing that SVs may be more likely to occur subsequent to an incident. ${ }^{10}$ RRT members should be appropriately trained and supported by the organization, be good listeners who express clear interest in the wellbeing of the affected individual, and avoid focusing on the details of the event. The word "rapid" is clearly operative within RRT, as involvement should ideally begin as early as possible, preferably within the first 24 hours following the event. Heiss points out that surgeons desire a readily available (24/7) peer support program that is confidential, and where reliable, predictable followup is assured to assist them throughout their experience. Furthermore, programs are successful when affected providers are interested in obtaining information regarding the SV experience and resources that might be available to them. ${ }^{12}$ This is a very personal process, and hence the experience for a given SV may not be the same as for others and requires individualized attention.

It is of importance for institutions to recognize the role of the system in incidents that occur. Too many healthcare personnel have voiced that their institution or organization have distanced themselves from the individual after an incident, creating further isolation and even renunciation. ${ }^{13}$ Institutions must be able to assure that the individual is not stigmatized as having committed a criminal act, but rather have a process for a systems-level analysis, with the anticipation that the organization will improve, and similar incidents would be avoided in the future. As noted earlier, all humans are fallible, and in the complex, highstakes healthcare environment, human error is always a risk and a negative outcome may be magnified. Malicious acts must be distinguished from a "mistake." This has been best exemplified in what Marx has described as a Just Culture, where human error is appropriately distinguished from a reckless action with unjustified risk-taking. As a result, the Just Culture emerges from a culture of "blame and shame. ${ }^{114}$ By promoting staff resiliency, wellness, systemlevel improvements, and patient safety, there is potential for reduced patient harm, with a positive domino effect of reduced staff burnout and SV syndrome.

Efforts to enhance a safety culture have been the foundation of highly reliable organizations, such as the military, nuclear energy organizations, and airlines, that is, high-risk environments. Historically, highly reliable organizations have demonstrated a far better safety record than healthcare by virtue of focusing on highly reliable communication, flattening hierarchy when possible, and the use of checklists and check-backs. Healthcare has only recently adopted the highly reliable organization philosophy, with the aim of reducing individual mistakes and subsequent patient harm. ${ }^{15,16}$ By assuring a Just Culture within a highly reliable organization, the Agency for Healthcare Research and Quality (AHRQ) definition of a Just Culture, one that "focuses on identifying and addressing systems issues that lead individuals to engage in unsafe behaviors, while maintaining individuals to engage in unsafe behaviors, while maintaining individual accountability by establishing zero tolerance for reckless behavior," can be a major goal that 
Koyle et al

is reachable. ${ }^{12,17}$ This Just Culture aim reduces the stigma of "shame and blame" felt by individuals, while augmenting patient safety efforts by encouraging the reporting of incidents, including "near misses." Such a culture shift may reduce overall stress within the organization, while providing enhanced communication, organizational learning, and improvement from past experience and best practices. ${ }^{12}$

\section{Conclusions}

As healthcare providers, the ever-increasing stresses of our chosen career may be imperceptible within the systems in which we work. Whereas the costs of an incident are wellunderstood at an administrative level, the effect on a hardworking, committed provider are too often overlooked. The wellness of healthcare providers has been an overlooked quality metric. The "wellness cost" to that clinician may be far more precious to colleagues within a system than the fiscal and litigious consequences. Medice Cura Te Ipsum, the Latin translation for "Physician heal thyself," the ancient Hebrew proverb in Luke 4:23, implies the physician must take care of himself/herself before attempting to heal others. For the SV and burnout sufferers, the road to recovery can be bumpy, and requires the assistance and support of those trained properly in personal and professional resilience. In an organization that promotes wellness, with a focus on reducing burnout and SV syndrome for those who provide care, when an incident occurs, the shame and isolation a colleague would have experienced can be replaced by a more positive, hopeful environment, in alignment with the quadruple aim.

\section{References}

1. James J. A new, evidence-based estimate of patient harms associated with hospital care. J Patient Saf 2013;9:122-8. https://doi.org/10.1097/PTS.0b013e3182948a69

2. Wu AW. Medical error: The second victim. The doctor who makes the mistake needs help too. BMJ 2000;320:726-7. https://doi.org/10.1136/bmi.320.7237.726

3. Institute of Medicine. To Err Is Human: Building a Safer Health System. Washing- ton, DC: The National Academies Press; 2000.

4. Bodenheimer T, Sinsky C. From triple to quadruple aim: Care of the patient requires care of the provider. Ann Fam Med 201412:573-6. https://doi.org/10.1370/afm.1713

5. Waterman AD, Garbutt J, Hazel $E$ et al. The emotional impact of medical errors on practicing physicians in the United States and Canada. Jt Comm J Qual Patient Saf 2007;33:467-76. https://doi.org/10.1016/S1553-7250(07)33050-X

6. Scott SD, Hirschinger LE, Cox KR, et al. The natural history of recovery for the healthcare provider "second victim" after adverse patient events. Qual Saf Health Care 2009;19:325-30. https://doi.org/10.1136/qshc.2009.032870

7. Hauk L. Understanding the second victim recovery process. AORN J 2018;107:4. https://doi.org/10.1002/aorn.12285

8. Shanafelt TD, Balch CM, Dyrbye L, et al. Special report: Suicidal ideation among American surgeons. Arch Surg 2011;146:54-62. hitps://doi.org/10.1001/archsurg.2010.292

9. Ozeke 0 , Aras $S$, Baser $K$, et al. Defensive medicine due to different fears by patients and physicians in geriatric atrial fibrillation patients and second victim syndrome. Int J Cardiol 2016; 212:251-2. https://doi.org/10.1016/i.i.jcard.2016.03.093

10. Scott SD, Hirschinger LE, Cox KR, et al. Caring for our own: Deploying a systemwide second victim rapid response team. Jt Comm J Qual Patient Saf 2010;36:233-40. https://doi.org/10.1016/ S1553-7250(10)36038-7

11. Hu YY, Fix ML, Hevelone ND, et al. Physicians' needs in coping with emotional stressors: The case for peer support. JAMA Surg 2012;147:212-7. https://doi.org/10.1001/archsurg.2011.312

12. Heiss K, Clifton M. The unmeasured quality metric: Burn out and the second victim syndrome in healthcare. Semin Pediatr Surg 2019;28:189-94. hrtps://doi.org/10.1053/i.sempedsurg.2019.04.011

13. Rassin M, Kanti T, Silner D. Chronology of medication errors by nurses: Accumulation of stresses and PTSD symptoms. Issues Ment Health Nurs 2005;26:873-86. https://doi.org/10.1080/01612840500184566

14. Marx D. Patient safety and the just culture. Obstet Gynecol Clin North Am 2019; 46:239-45. https://doi.org/10.1016/i.ogc.2019.01.003

15. Cohen DL, Stewart KO. The stories clinicians tell: Achieving high reliability and improving patient safety. Perm J 2016;20:85-90.

16. Tsao K, Browne M. Culture of safety: A foundation for patient care. Semin Pediatr Surg 2015;24:283-7. https://doi.org/10.1053/i.sempedsurg.2015.08.005

17. Agency for Healthcare Research and Quality (AHRQ). Patient safety primers, safety culture. http://psnet.ahrq.gov/primer.aspx?primerlD=5; 2012.

Correspondence: Dr. Martin A. Koyle, SickKids, Toronto, ON, Canada; Martin.koyle@sickkids.ca 